\title{
Computational Fluid Dynamics Methods and Their Applications in Medical Science
}

\author{
Wojciech Kowalewski ${ }^{1}$, Magdalena Roszak ${ }^{2}$, Barbara Kołodziejczak ${ }^{2}$, \\ Anna Ren-Kurc ${ }^{3}$, Andrzej Bręborowicz ${ }^{4}$ \\ ${ }^{1}$ Faculty of Geographical and Geological Science, Adam Mickiewicz University in Poznan, \\ Poland \\ 2 Department of Computer Science and Statistics, Poznan University of Medical Sciences, \\ Poland \\ 3 Faculty of Mathematics and Computer Science, Adam Mickiewicz University in Poznan, \\ Poland \\ 4 Department of Pathophysiology, Poznan University of Medical Sciences, Poland
}

\begin{abstract}
As defined by the National Institutes of Health: "Biomedical engineering integrates physical, chemical, mathematical, and computational sciences and engineering principles to study biology, medicine, behavior, and health". Many issues in this area are closely related to fluid dynamics. This paper provides an overview of the basic concepts concerning Computational Fluid Dynamics and its applications in medicine.
\end{abstract}

\section{Introduction}

Approximately $65 \%$ of the human body is composed of water. Fluids are responsible for a considerable part of its physiology. The process of breathing is related to the passage of air (i.e. gas) to the lungs and complex chemical processes that govern the flow of its components through the human organism. Due to the similarity in physical characteristics (dynamics of motion, in particular), liquids and gases are classified as a single group, which is referred to as fluids. The aim of this paper is the reasonably comprehensive presentation of concepts the knowledge of which is required in order to conduct computer-assisted analyses of the behavior of fluids in various conditions. Such analyses, in general, are affiliated with the field known as computational fluid dynamics (CFD). Therefore, some fragments may be found complicated by readers, but at the same time, they emphasize the demand for experienced specialists in teams that study specific cases. 
Significant conclusions that address this issue are presented in the last chapter. Such analyses have been widely employed in medical research (Chandran et al., 2012; Pozrikidis, 2010; Rubenstein et al., 2015; Spagnolie, 2015; Tu et al., 2013, 2015). For example:

- Macrocirculation

- mechanics of blood flow through the heart with the values of the systolic and diastolic pressure as parameters - a description based on Navier-Stokes equations

- blood flow through veins and arteries with an allowance for resistance; Windkessel effect with pulsation described by the HagenPoiseuille formulas

\section{- Microcirculation}

- blood flow in aneurysms in accordance with Bernoulli's principle

- lymphatic system with osmotic transport and muscles as the pumping mechanism

- gas exchange in the respiratory system modeled in accordance with the Boyle-Mariott law.

\section{Classification of Fluids}

In standard physics, four states of matter are considered: solids, liquids, gases and plasma. The first two belong to the group of condensed bodies, which is characterized by strong intermolecular interactions, which in turn result in the intensive coordination of atom positioning (in case of solid bodies, in a large area). Gases and plasma form the group of uncondensed bodies, in which intermolecular interactions are weak enough to enable the independent movement of molecules, at least for short distances. Solid bodies and liquids are associated with the notion of a thermodynamic phase, i.e. the part of the physical system that is uniform with respect to both physical and chemical properties. Generally, in solid bodies and liquids, several thermodynamic phases may occur concurrently, being separated by interfacial surfaces (phase borders), on which gradual changes of properties take place.

In the case of liquids, multiphase systems occur in the form of heterogeneous mixtures. Broadly speaking, we can also differentiate homogeneous mixtures, known as proper solutions, which comprise single-phase systems. Improper solutions are multiphase and thus classified as heterogeneous mixtures. In general, the classification of heterogeneous mixtures, especially the distinction between improper and proper solutions, is ambiguous and is highly dependent on the scale of measure. As such, it sometimes requires subtle analyses. 
Heterogeneous mixtures have two or more thermodynamic phases. Usually, one of them is treated as the dispersing phase (a type of solvent, which is commonly continuous), while others are considered as dispersed phases (solutes, which for the most part form a material that is more or less refined). Mixtures are generally divided into one of three groups, depending on the magnitude of the dispersed phase:

- Solutions - the dispersing phase is referred to as a solvent, while the dispersed phase is a solute. Molecules of the dispersed phase are invisible, and their diameter is smaller than $10^{-9} \mathrm{~m}$, i.e. smaller than $1 \mathrm{~nm}$ (similar to the dimensions of molecules of a chemical compound).

- Colloids - mixtures in which the diameter of dispersed phase molecules falls within the range of $\left[10^{-9} \mathrm{~m}, 10^{-6} \mathrm{~m}\right]$, i.e. $1 \mathrm{~nm}-1 \mu \mathrm{m}$.

- Suspensions - a solution in which the diameter of dispersed phase molecules is larger than $10^{-6} \mathrm{~m}$, i.e. larger than $1 \mu \mathrm{m}$.

Examples

- In solutions, in cases where gas is the dispersing phase, the only possible state of aggregation for the dispersed substance is gas as well. Dry, clean air is the obvious example thereof, since nitrogen $\left(N_{2}\right)$ acts as the solvent, while oxygen $\left(\mathrm{O}_{2}\right)$, argon $(\mathrm{Ar})$, carbon dioxide $\left(\mathrm{CO}_{2}\right)$, neon $(\mathrm{Ne})$, helium $(\mathrm{He})$, methane $\left(\mathrm{CH}_{4}\right)$, krypton $(\mathrm{Kr})$, hydrogen $\left(\mathrm{H}_{2}\right)$ and xenon $(\mathrm{Xe})$ are the dissolved substances.

- Human blood is a more complex mixture. It may be perceived as the suspension of cell elements (solids as a state of matter) in the blood plasma (liquid as a state of matter). Volume proportions of cell elements and blood plasma are $45.7 \%$ and $54.3 \%$, respectively. Cell elements comprise:

- red blood cells (erythrocytes) - they constitute $99.7 \%$ of cell elements and assume the form of biconcave disks of $2.5 \mu \mathrm{m}$ in thickness and $8 \mu \mathrm{m}$ in diameter.

- white blood cells (leukocytes) - they represent $99.7 \%$ of cell elements and form spheres of diameters ranging from 20 to $100 \mu \mathrm{m}$. Among leukocytes, we can distinguish granulocytes (eosinophils, basophils and neutrophils), lymphocytes and monocytes.

- platelets (thrombocytes) - they account for $99.7 \%$ of cell elements and take the form of ellipsoids with the length of their axes amounting to $1.5 \mu \mathrm{m}$ and $4 \mu \mathrm{m}$, respectively. Blood plasma is already a solution, with water being the solvent and the list of dissolved substances including, among others: sodium ions, chlorides, potassium, magnesium and calcium ions, phosphates, and proteins - albumins and globulins $\left(\alpha_{1}, \alpha_{2}, \beta, \gamma\right)$. 
Wojciech Kowalewski et al.

\section{Equations}

Fluids can be analyzed on three different levels of accuracy. The development of a mathematical model suitable for the given problem is essential. Significant theoretical elements of these three approaches are presented below. Some details and the occasional presence of mathematical formalism will require the readers who wish to fully understand the presented material (despite the presentation being rather synthetic) to reach for complementary readings. Others shall realize what problems are to be tackled in the analysis of fluid behaviors and what kind of specialists need to be asked for cooperation.

\section{Macroscopic Model}

This approach involves the employment of equations and tools of classical continuum mechanics. This description is epitomized by the NavierStokes equation. Continuity of matter is comprehended here as the omission of its molecular structure. What indicates the potential of such approximation is the Knudsen number $(\boldsymbol{K} \boldsymbol{n})$. In practice, fluids with a Knudsen number lower than $\mathbf{0 . 0 1}$ may be treated as being composed of continuous matter.

Let us assume that $\mathcal{D} \subset \mathbb{R}^{3}$ denotes the volume within which the fluid is analyzed. In different moments in time, the fluid may be located in various areas of the volume $\mathcal{D}$. It may also have a static location. Let us consider a certain value $H$ (variable of interest), scalar or vector, in the region $\mathcal{D}$. It produces in $\mathcal{D}$ the scalar or vector field, respectively. In various $\mathcal{D}$ points, this field may have different values. Furthermore, it may change in time at any point. It must be emphasized that the field which has been defined in such fashion may be perceived in two ways. On the one hand, it may be considered as the property of the region - then, points of the region are treated as fixed. On the other hand, we may observe only those fragments of $\mathcal{D}$ which contain fluid, tracking the movement of molecules of this fluid. In such a case, locations (related to the fluid) in $\mathcal{D}$ are treated as changing in time. Now, let us describe this issue in a slightly more precise manner by presenting fundamental principles that govern flow dynamics in a more formal depiction. We have decided to pass the question of turbulent flows over, due to their rather insignificant applicability in medicine.

Let us assume that $\mathbf{r}(t)=(x(t), y(t), z(t)) \in \mathcal{D}$ and $Y$ is one of the fields $\mathbb{R}, \mathbb{R}^{2}, \mathbb{R}^{3}$. We learn that $H$ is the following function:

$$
H:\left[T_{0}, T_{1}\right] \times \mathcal{D} \rightarrow Y, \quad(t, \mathbf{r}) \rightarrow H(t, \mathbf{r}),
$$

where $\left[T_{0}, T_{1}\right]$ is the analyzed time interval. 
The derivative of this function relative to time is known as the material derivative. Accordingly, the material derivative is calculated in the same fashion as the composite function derivative.

$$
\begin{aligned}
\frac{d H}{d t}(t, \mathbf{r}(t)) & =\left(\frac{\partial H}{\partial t}+\frac{\partial H}{\partial x} \frac{d x}{d t}+\frac{\partial H}{\partial y} \frac{d y}{d t}+\frac{\partial H}{\partial z} \frac{d z}{d t}\right)(t, \mathbf{r}(t)) \\
& =\left(\frac{\partial H}{\partial t}+\vec{v} \cdot \nabla H\right)(t, \mathbf{r}(t)),
\end{aligned}
$$

as $\vec{v}(t, r(t))=\mathbf{r}^{\prime}(t)$

The element is $\frac{\partial H}{\partial t}$, called the local derivative, and it represents changes of $H$ in the precisely specified point, through which other elements flow. If the derivative is zero, then the field $H$, which is otherwise non-stationary, is stationary. Stationarity is the feature of the region, not the fluid, since it only denotes that the field, in the specified point, remains unchanged in time. Nevertheless, if the field is bound with a fluid molecule that is present at this point at the given moment, then it may change the value of this field after moving to another point.

Element $\vec{v} \cdot \nabla H$ is known as the convective derivative - it shows the change of $H$ in time as the result of the fluid element moving at the speed of $\vec{v}$ from a point with a given value of $H$ to another point with a different value. It may also be interpreted as the observation of the field by an observer who is situated at a given point and moves at the velocity $\vec{v}$. If the convective derivative is non-zero in a given point of the field, then such a field is heterogeneous. Otherwise, if it is zero in the entire field, then it is regarded as homogeneous.

In order to analyze the behavior of fluids on a macroscopic level, we can generally adopt one of two approaches:

- Lagrangian approach - the behavior of a chosen fluid molecule (position, velocity, etc.) in time is examined, which enables the tracking of fluid paths and provides us with the direct overview of its dynamics (Figure 1).

- Eulerian approach - in this case, we focus on the analysis of points (stationary, i.e. their position does not change in time!) in space, in which the fluid is contained, by defining the change of their properties over time - the location of the examined point is fixed, but various fluid molecules of distinct properties may flow through this point at different moments in time (velocity etc.). This approach places emphasis on properties of the field of the fluid without, obviously, revealing its dynamics (Figure 2). 
Wojciech Kowalewski et al.

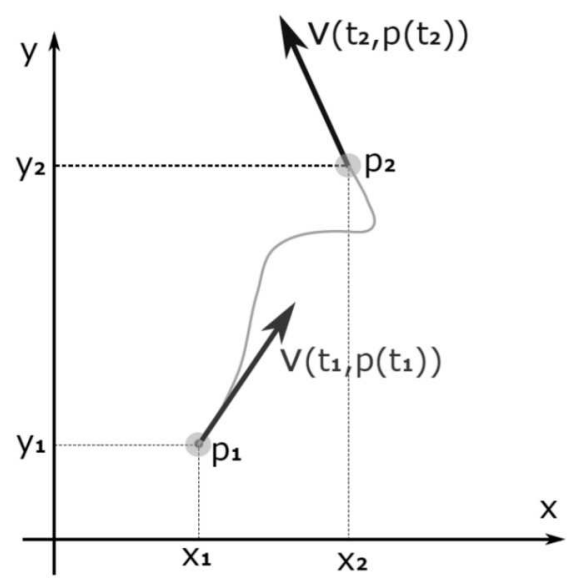

Figure 1. Lagrangian approach - the fluid molecule in time $t_{1}$ is situated at point $p_{1}=p\left(t_{1}\right)$ and moves at the velocity $\vec{v}\left(t_{1}, p\left(t_{1}\right)\right)$. Then, it moves between moments $t_{1}$ and $t_{2}$ along the path, reaching in time $t_{2}$ point $p_{2}=p\left(t_{2}\right)$ at the velocity $\vec{v}\left(t_{2}, p\left(t_{2}\right)\right)$

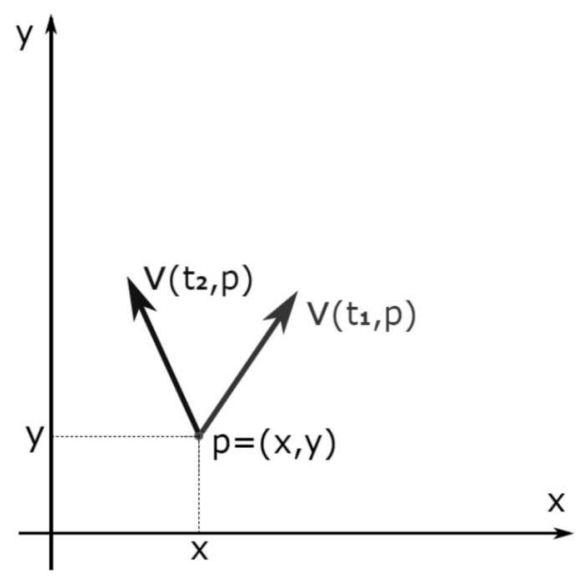

Figure 2. Eulerian approach - at the given point $p=(x, y)$ of the fluid domain, vectors of velocity in two distinct moments in time are shown: $t_{1}$ and $t_{2}$

In Figure 1, it is clearly visible that according to the Lagrangian approach, the hypothetical point $p_{1}$ is not parameterized with time from the perspective of the fluid domain, while time is the parameter of the location of the determined fluid molecule $p\left(t_{1}\right)$, which is being tracked.

The flow of the fluid is incompressible, provided that none of its moving parts change their density in time. The fluid is incompressible if the density of each molecule is constant in time. 
Let us consider the value $\varphi$ (vector or scalar) at the moment $t$ at the point $\mathbf{r}=(x, y, z) \in V \subset \mathbb{R}^{3}$ in units of the volumetric density, i.e. $[\varphi]=j /$ $m^{3}$, where $j$ is the unit of measure $\varphi$ (Spiegelman, 2000). The boundary of volume $V$ is formed by surface $S=\partial V$ (Figure 3), meaning the perpendicular vector to $\partial V$ is well defined at each point $\mathbf{s} \in \partial V$. This vector is marked with $\overrightarrow{d S}$, being combined with the differential surface $d S:=d S(\mathbf{s})$ through the normal vector $\vec{n}$, i.e. a unitary vector that is perpendicular to the surface at the given point: $\overrightarrow{d S}=d S \cdot \vec{n}$. Pursuant to common convention, the sense of a vector that is perpendicular to the closed surface is directed towards outside $\partial V$. The $\operatorname{sum} \varphi$ in the volume $V$ is represented by an integral

$$
\varphi_{V}(t)=\int_{V} \varphi(t, \mathbf{r}) d V, \quad\left[\varphi_{V}\right]=j
$$

Note. It is assumed that the value $\varphi$ has been properly specified in the analyzed space, i.e. if required, the space is not a vacuum and thus contains molecules of a certain medium.

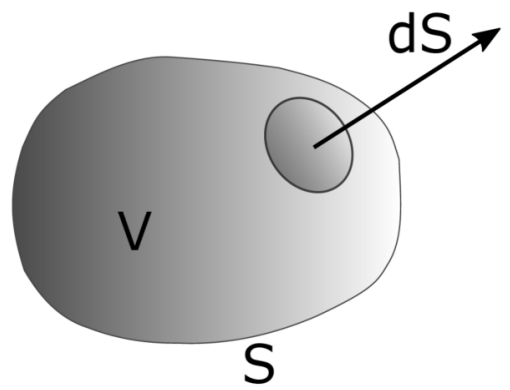

Figure 3. Volume $V$ is limited with the boundary $S$ that is directed toward the outside

Let us examine the rate at which value $\varphi$ changes in volume $V$, i.e.

$$
\frac{d}{d t} \varphi_{V}(t)=\frac{d}{d t} \int_{V} \varphi(t, \mathbf{r}) d V, \quad\left[\frac{d}{d t} \varphi_{V}(t)\right]=\frac{j}{s}
$$

The change of value $\varphi$ in volume $V$ may generally result from twofold variations: those that originate in volume $V$ and those that develop from the interaction of volume $V$ with the environment. The latter are detected by the boundary surface $\partial V$. In both types, factors that increase or decrease the number of values $\varphi$ in volume $V$ may occur (Figure 4). 


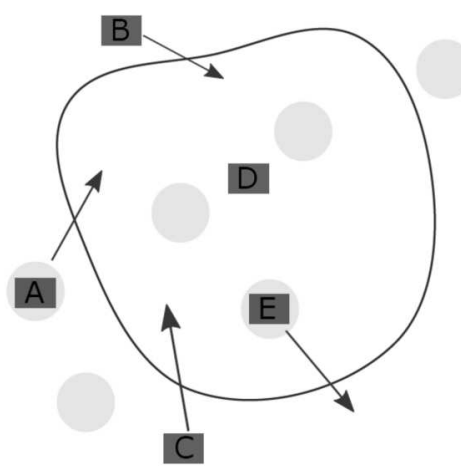

a)

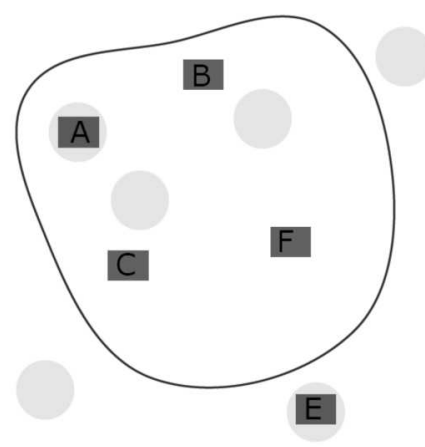

b)

Figure 4. Transportation diagram for the value $\varphi$. Medium molecules are marked with spheres, and values $\varphi$ are marked with rectangles. Part b) represents later

1. volume $V$ as the generator of measure $\varphi$ - rectangle $\mathrm{F}$

2. volume $V$ as the annihilator of measure $\varphi$ - rectangle D

3. stream of value $\varphi$ through the surface $\partial V$ into (the exterior of) volume $V$

1. value $\varphi$ transported to (from) volume $V$ with the aid of medium molecules - rectangle A (E)

2. value $\varphi$ transported to (from) volume $V$ without medium molecules - rectangle $\mathrm{B}$ and $\mathrm{C}$

Then, the equation that illustrates the sum of these behaviors takes the following form:

[General conservation law]

$$
\frac{\partial}{\partial t} \varphi(t, \mathbf{r})-H(t, \mathbf{r})+\nabla \cdot(\vec{F}+\varphi \vec{v})(t, \mathbf{r})=0,
$$

where $H$ is the resultant of emission and annihilation, whereas $F$ is the resultant of streams without molecules. Let us now consider certain particular cases of the general conservation law.

1. Let us assume that $\varphi$ denotes mass density. It is commonly defined through $\rho(t, \mathbf{r})$. Then $[\rho]=k g / \mathrm{m}^{3}$. Naturally, the source term is $H=0$ since mass can neither vanish nor be created unaided. Moreover, the term is also $F=0$ since mass can move only with the aid of medium molecules (which, for the most part, are mass themselves). We eventually arrive at the formula for the law of the conservation of mass, also known as the equation of continuity (with arguments of variables having been intentionally omitted). 
Computational Fluid Dynamics Methods and Their Applications...

[Equation of continuity]

$$
\frac{\partial}{\partial t} \rho+\nabla \cdot(\rho \vec{v})=0
$$

By using the dependence

$$
\nabla \cdot(s \vec{w})=\vec{w} \cdot \nabla s+s \nabla \cdot \vec{w}
$$

of the divergence of scalar $s$ and vector $\vec{w}$, we shall get

$$
\frac{\partial \rho}{\partial t}+\vec{v} \cdot \nabla \rho+\rho \nabla \cdot \vec{v}=0
$$

The first term is a local derivative, whereas the other one is a derivative of the density convection. In total, in accordance with the equation (2), they form a material derivative $d \rho / d t$. So the equivalent form of the equation (7) is

$$
\frac{d \rho}{d t}+\rho \nabla \cdot \vec{v}=0 .
$$

Three canonical versions of density fields are as follows:

- Stationary, compressible density field; that is, stationary and compressible flow. It is represented by the zeroing condition of the local derivative, while density may be different in various points of the field (heterogeneity). Therefore, the equation of the stationary, compressible flow, as the variation of equation (6), takes the following form:

$$
\nabla \cdot(\rho \vec{v})=0 .
$$

- Stationary, incompressible density field; that is, stationary and incompressible flow. It is represented by the zeroing condition of the local derivative and equal density in the entire field (homogeneity). Thus, based on formula (8), the equation of the stationary, incompressible flow is constructed as follows:

$$
\nabla \cdot \vec{v}=0 .
$$

- Non-stationary, compressible density field; that is, non-stationary and compressible flow. The field is variable both in time and space (heterogeneity). The equation of the stationary, compressible flow is naturally the equation (7). 
Wojciech Kowalewski et al.

As results from the law of conservation of mass, for the fluid of volume $V$

$$
\nabla \cdot \vec{v}=\frac{1}{V} \frac{d V}{d t}
$$

so the divergence of velocity denotes the rate of change of the specific volume of the fluid in motion.

2. Let us assume that $\varphi=\rho c_{P} T$, where $c_{P}$ denotes specific heat with the pressure determined as $\left(\left[c_{P}\right]=J / m^{3} K\right)$. Thermal flux has two components: $\vec{F}=-k \nabla T$, where $k$ denotes thermal conductivity and the transportation term related to $\varphi$. By gathering all types of heat sources and heat sinks of the analyzed volume in $H$, we shall obtain the equation of the conservation of energy.

[Equation of the conservation of energy]

$$
\frac{\partial}{\partial t} \rho c_{P} T+\nabla \cdot\left(\rho c_{P} T \vec{v}\right)=\nabla \cdot(k \nabla T)+H
$$

3. Let us assume that $\varphi=\rho \vec{v}$ (momentum on the unit of volume), vector of the source term $\vec{H}=\rho \vec{g}$ (gravity force on the unit of volume), while the stream $\vec{F}$ denotes the surface force acting on the unit of the fluid's surface (usually referred to as the surface tension and denoted $\vec{\sigma}$ ). Surface tension is commonly divided into perpendicular components (scalars), in other words pressure (marked as $p$ ), and tangential components (vector) (in classic cases known as viscosity). This leads us to the equation of conservation of momentum in the NavierStokes form:

[Navier-Stokes equation]

$$
\frac{\partial}{\partial t} \vec{v}+\vec{v} \cdot \nabla \vec{v}=\frac{1}{\rho} \nabla p+v \nabla^{2} \vec{v}+\vec{g},
$$

where $v$ is the dynamic viscosity, while $\nabla \vec{v}$ is the so-called generalized gradient. Such form of the Navier-Stokes equation indicates that the fluid belongs to the group known as incompressible Newtonian fluids, i.e. tangential stress is the linear function of nondilatational strain matrices. Dynamics of non-Newtonian fluids are considerably more difficult to describe - these fluids are exemplified by human blood.

\section{Microscopic Model}

The conception of this model is based on the description derived from the kinetic theory of matter. It assumes that matter is comprised of randomly moving molecules with space between them. In this case, the natural 
measure of quantity is the mole (the amount of matter that contains the number of molecules equal to Avogadro's number $N_{A}$ ), from which the notion of molecular mass is derived (denoted $M_{m o l}$ ), i.e. mass of a single mole of the given matter. While considering matter of volume $V$ and mass $M$, we can see that the mole number of this matter is $n=M / M_{m o l}$, and the total number of molecules in this volume amounts to $N=n N_{A}$. Thus, the volume occupied by a single molecule of this matter equals $V / N$. By reducing this volume conceptually to a single dimension and determining the density of the analyzed matter through $\rho=M / V$, we can define the length of the molecular separation as:

$$
L_{m o l}=\left(\frac{V}{N}\right)^{\frac{1}{3}}=\left(\frac{M_{m o l}}{\rho N_{A}}\right)^{\frac{1}{3}}, \quad\left[L_{m o l}\right]=n m .
$$

For solids and liquids that are dense in their natural forms, the length of molecular separation is comparable to the size of molecules, i.e. it equals one angstrom. For example, in the case of iron, it is $L_{m o l} \approx 0.23 \mathrm{~nm}$, while for water this value is rounded off to $0.31 \mathrm{~nm}$. Gases are composed of molecules that are considerably looser. For example, in normal conditions, an ideal gas (pressure: 1 atmosphere, temperature: $20^{\circ} \mathrm{C}$ ) is characterized by $L_{m o l} \approx$ $3.4 \mathrm{~nm}$. This implies that in ideal gases the empty space is approx. 1000 times larger than the volume of the molecules contained therein.

For mixtures, the length of molecular separation is defined by the mean molar mass of respective phases. The employment of molecular mass in the definition of $L_{m o l}$ implies that molecular masses of consecutive phases will be scaled with the amount of moles, determined in terms of percentage, in these phases. Let us use $M_{i}^{m o l}$ and $n_{i}$ to denote, respectively, molecular mass and the mole number for the phase $i$. The total mole number in the mixture amounts to $n=\Sigma_{i} n_{i}$, while the total mass of the mixture equals $M=\Sigma_{i} n_{i} M_{i}^{\text {mol }}$. Then, the mean molar mass

$$
M_{m o l}=\frac{M}{n}=\sum_{i} \frac{n_{i}}{n} M_{i}^{m o l} .
$$

For example, in case of air, considered in simple terms as the mixture of nitrogen, oxygen and argon, the mean molecular mass amounts to $28.95 \mathrm{~g} / \mathrm{mol}$.

On the microscopic level, we do not consider gravitational forces, but intermolecular forces and (less often in discussing dynamics) intramolecular forces, i.e. chemical bonds. Fundamental features that make them distinguishable include the higher strength of chemical bonds and the resulting stability of their interactions. In general, chemical bonds are divided into 
three distinct groups, depending on the remainder of electronegativity between bonded atoms: for the remainder below 0.4, covalent nonpolar bonds are created, whereas for the values within the range of $0.4-1.7$, covalent polar bonds are formed; if the remainder exceeds 1.7, atoms are bonded with ionic bonds. Furthermore, we also distinguish hydrogen bonds, the nature of which differs from the standard electron exchange and which are significantly weaker than covalent and ionic bonds. Bonding of dipoles of water molecules constitutes a classic example in this case. The hydrogen bond is considered within the cross-section of the group of bonds and the group of intermolecular forces. In the second group, we discern ion-ion interactions, which are stronger than hydrogen bonds, and van der Waals forces, which are weaker but more common. The latter are additionally divided into permanent dipole-permanent dipole interactions (known as Keesom forces, e.g. hydrogen chloride molecule $\mathrm{HCl}$ ), induced dipole-induced dipole attraction, known as Debye interaction, and temporary dipole-induced dipole interactions, referred to as London forces (the only interactions between molecules of noble gases).

The foundation of the microscopic modeling of fluids is the equation of state, which shows reciprocal dependencies between parameters of the system that is analyzed as a thermodynamic system. Rare gases can be approximated with the equation of state of an ideal gas, in which we consider only elastic collisions of chaotically moving molecules (absence of classical intermolecular interactions). This equation is known as the Clapeyron equation, and it delivers the dependency between gas pressure $p$, its volume $V$, temperature $T$ and the number of moles $n$ :

$$
p V=n R T
$$

where $R$ is the universal gas constant: $R=N_{A} k_{B}, N_{A}$-Avogadro's number, $k_{B}$ - Boltzmann constant, $R=8.314 \mathrm{~J} /(\mathrm{mol} \cdot \mathrm{K})$ Its more accurate version is provided in the Van der Waals equation of state, which takes into consideration the molecular volume $V_{m}=V / n$, the volume of the mole of gas molecules $b$ as well as reciprocal interactions of molecules (constant $a$ ):

$$
\left(p+\frac{a}{V_{m}^{2}}\right)\left(V_{m}-b\right)=R T .
$$

The movement of molecules that are considered in microscopic modeling as separate masses is calculated through the second law of motion, with collisions allowed for, whereby the intermolecular force is often computed as the derivative of the potential energy (denoted $U$ ). Thus, the typical 
system of equations for dynamics in a simple system of $N$ atoms assumes the following form:

$$
m_{i} \frac{d^{2} \vec{r}_{i}}{d t^{2}}=f_{i}, \quad f_{i}=\frac{\partial U}{\partial r_{i}}, \quad i=1, \ldots, N .
$$

In practical calculations of interactions between atoms or molecules, a convenient approximation of potential energy is frequently employed through the Lennard-Jones potential, which separates attractive and repulsive interactions. The former are in this approximation proportional to $R^{-6}$, while the latter remain proportional to $R^{-12}$, where $R$ denotes the distance between atoms/molecules. Then

$$
U_{L J}=4 \varepsilon\left[\left(\frac{\sigma}{R}\right)^{12}-\left(\frac{\sigma}{R}\right)^{6}\right],
$$

where $\sigma$ denotes the distance for which the interaction energy is 0 , while $\varepsilon$ is the depth of the potential well $\left(U_{L J}\right.$ achieves its minimum value equal to $-\varepsilon$ for $R=1.22 \sigma)$.

\section{Mesoscopic Model}

This approach fills the gap between the macroscopic and microscopic description, in an attempt to apply the law of the kinetic theory of matter for problems related to flows on macroscopic scales. The key here is the Boltzmann transport equation and the distribution function contained therein (denoted $f$ ), which determines the number of (mostly abstract) molecules moving at the moment $t$ in volume $d \mathbf{r}$ (which includes point $\mathbf{r}$ ), with the velocity $\vec{v}=\left[v_{x}, v_{y}, v_{z}\right]$. Thus, such amount of molecules is determined by the following expression:

$$
f(\mathbf{r}, \vec{v}, t) d \mathbf{r} d \vec{v} .
$$

What is intriguing here is the fact that potential values $d \mathbf{r}$ and $d \vec{v}$ are differentials from the mathematical point of view, but the boundary crossing to 0 is not substantiated in terms of physics since the uncertainty principle prohibits such situations in quantum physics, and in classical physics such a crossing brings the loss of continuity and smoothness of function $f$, while these exact properties are useful in the mesoscopic approach. Thus, $d \mathbf{r}$ is comprehended here as large enough to contain a large number of molecules and at the same time small enough to avoid classification as a macroscopic case. For example, if $d \mathbf{r}$ amounts to $10^{-12} \mathrm{~m}$, the diameter of volume reaches $10^{-4}$, making it possible for $d \mathbf{r}$ to con- 
tain enough molecules for fine suspensions. The Boltzmann transport equation (also referred to as the Boltzmann kinetic equation) is similar in its construction to dynamic equations in the macroscopic approach - it determines the material derivative of the distribution function. That is, it simply measures the rate at which the amount of molecules of specific properties changes. However, in this case, function $f$ is dependent on three additional variables that create the vector $\vec{v}$. Therefore, by denoting the vector of acceleration with $\vec{a}=\vec{v}^{\prime}$ the material derivative comprises three blocks. By assuming that the only sources of changes in the molecule distribution are collisions and by denoting the integral collision with $B(f, f)$ in the phase volume $d \mathbf{b} d \vec{v}$, we obtain the basic form of this equation.

[Boltzmann Transport Equation]

$$
\frac{\partial}{\partial t} f+\vec{v} \cdot \nabla_{\mathbf{r}} f+\vec{a} \cdot \nabla_{\vec{v}} f=B(f, f) .
$$

When collisions in the fluid do not occur (the right side of the Boltzmann Transport Equation is zero), then the fluid is incompressible.

\section{Numerical Methods}

In practice, even the simplest variants of the presented equations do not have analytic solutions. Therefore, the only viable solution is to solve them with the aid of computers. This leads us straight into the vast field of numerical methods, where even the fragment concerning the issue of solving fluid equations is colossal and contains hundreds of specialized algorithms. They can be classified in a variety of ways, but we have decided to present the classification that is based on the division which is used for classifying equations. Then, two examples of such methods are presented along with the indication of their applicability in medical research.

\section{Classification of Methods of Computer-Assisted Fluid Dynamics} Macroscopic models

- Grid methods

1. Euler description

- Finite Difference Method (FDM)

- Finite Volume Method (FVM)

- Volume of Fluid (VOF)

- Level Set Method (LSM) 
Computational Fluid Dynamics Methods and Their Applications...

2. Lagrangian description

- Finite Element Method (FEM)

- Non-grid methods

- Direct Simulation Monte Carlo (DSMC)

- Smoothed particle hydrodynamics (SPH)

Microscopic models

- Non-grid methods

- Direct Simulation Monte Carlo (DSMC)

- Molecular Dynamics (MD)

- Particle Mesh (PM)

- Particle ParticleParticle Mesh (PPPM)

Mesoscopic models

- Lattice Boltzman Method (LBM)

\section{Diffusion Equation and the FVM Method}

Diffusion is the process of spontaneous propagation of molecules or energy in each medium, the consequence of chaotic collisions between molecules of diffusing substances or with other molecules contained in the environment (Figure 5).
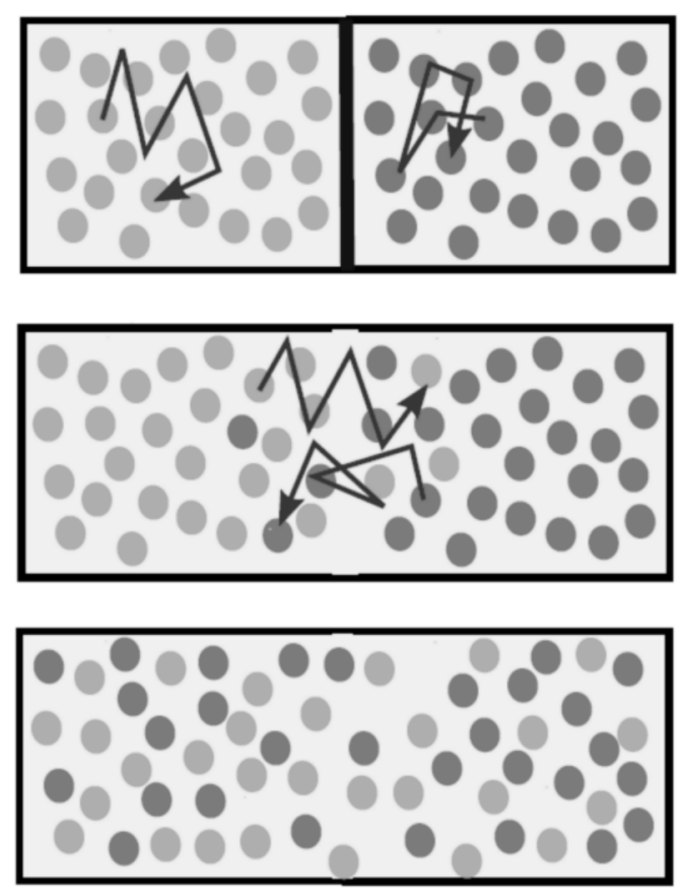

Figure 5. Diffusion of molecules of two types after the removal of an obstacle 
Wojciech Kowalewski et al.

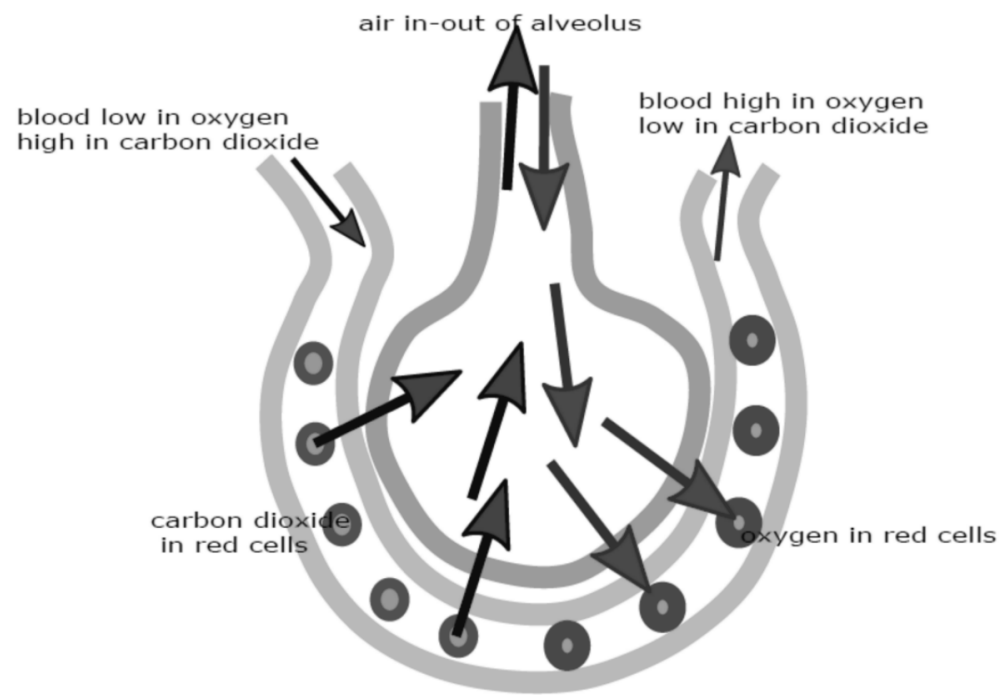

Figure 6. Diffusion of oxygen and carbon dioxide in the lungs

One of the most evident examples of this process in the human organism is the diffusion of oxygen and carbon dioxide in the lungs (Figure 6).

The diffusion equation may be treated as a special case of the NavierStokes equation - while determining concentration of a substance through $\varphi(\mathbf{r}, t)\left(\left[\mathrm{mol} / \mathrm{m}^{3}\right]\right)$, it takes the following form:

$$
\frac{\partial \varphi}{\partial t}=D \nabla^{2} \varphi
$$

The diffusion coefficient $D$ is bound by the Stokes-Einstein law with the dynamic viscosity $\nu$, which is included in the Navier-Stokes equation, but its applicability has been confirmed for large molecules only.

The Finite Volume Method (Versteeg et al., 2007), as other numerical methods, discretizes the equation domain by defining the infinite amount of computational points within it. In the case of the FVM, the volume $V$, analyzed in the finite time interval $\left[t_{0}, t_{1}\right]$, is divided into a finite number of control volumes $V_{p}$ and a finite number of time intervals $[t, t+\Delta t]$ (Figure 7 ). Then, we need to integrate the equation (19) on the elements of this division

$$
\int_{t}^{t+\Delta t} \int_{V_{p}}\left(\frac{\partial \varphi}{\partial t}-D \nabla^{2} \varphi\right) d V d t=0
$$




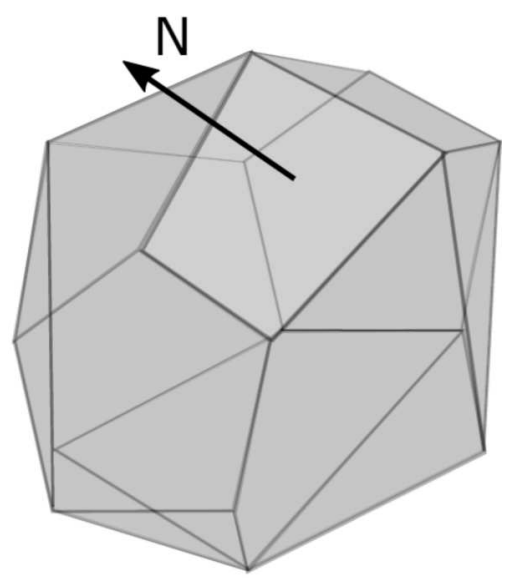

Figure 7. Example of the control volume topology

The integral from the local derivative is approximated through the difference quotient to the derivative under the integral in the time interval $\left[t_{n}, t_{n+1}\right]$.

$$
\int_{t}^{t+\Delta t} \int_{V_{p}} \frac{\partial \varphi}{\partial t} d V d t \approx V_{p} \frac{\varphi^{n+1}-\varphi^{n}}{\Delta t}
$$

The integral from the diffusion term is first transcribed to the integral over the surface $\partial V_{p}$, which limits volume $V_{p}$ :

$$
\int_{t}^{t+\Delta t} \int_{V_{p}} D \nabla^{2} \varphi d V d t=\int_{t}^{t+\Delta t} \int_{\partial V_{p}} D \nabla \varphi \cdot \overrightarrow{d S} d S
$$

and then the surface integral is approximated with the standard numerical method, i.e. one of the numerical quadratures, e.g.

$$
\int_{t}^{t+\Delta t} \int_{\partial V_{p}} D \nabla \varphi \cdot \overrightarrow{d S} d S \approx \sum_{1}^{N} D_{i}(\nabla \varphi)_{i} \cdot N_{i}
$$

where index $i$ denotes the successive sample of parameters under the integral. As an example (Figure 8), the mixing of two fluids of different diffusion coefficients is presented. In the central figures, the visualization of the velocity field is also presented. In the last figure, the movement paths for certain molecules, which more or less correspond to the movement phase shown in the penultimate figure, are visualized. The visualization was made in the OpenFoam environment (see www.openfoam.com), which implements, among others, the FVM method (Marić et al., 2014). 
Wojciech Kowalewski et al.
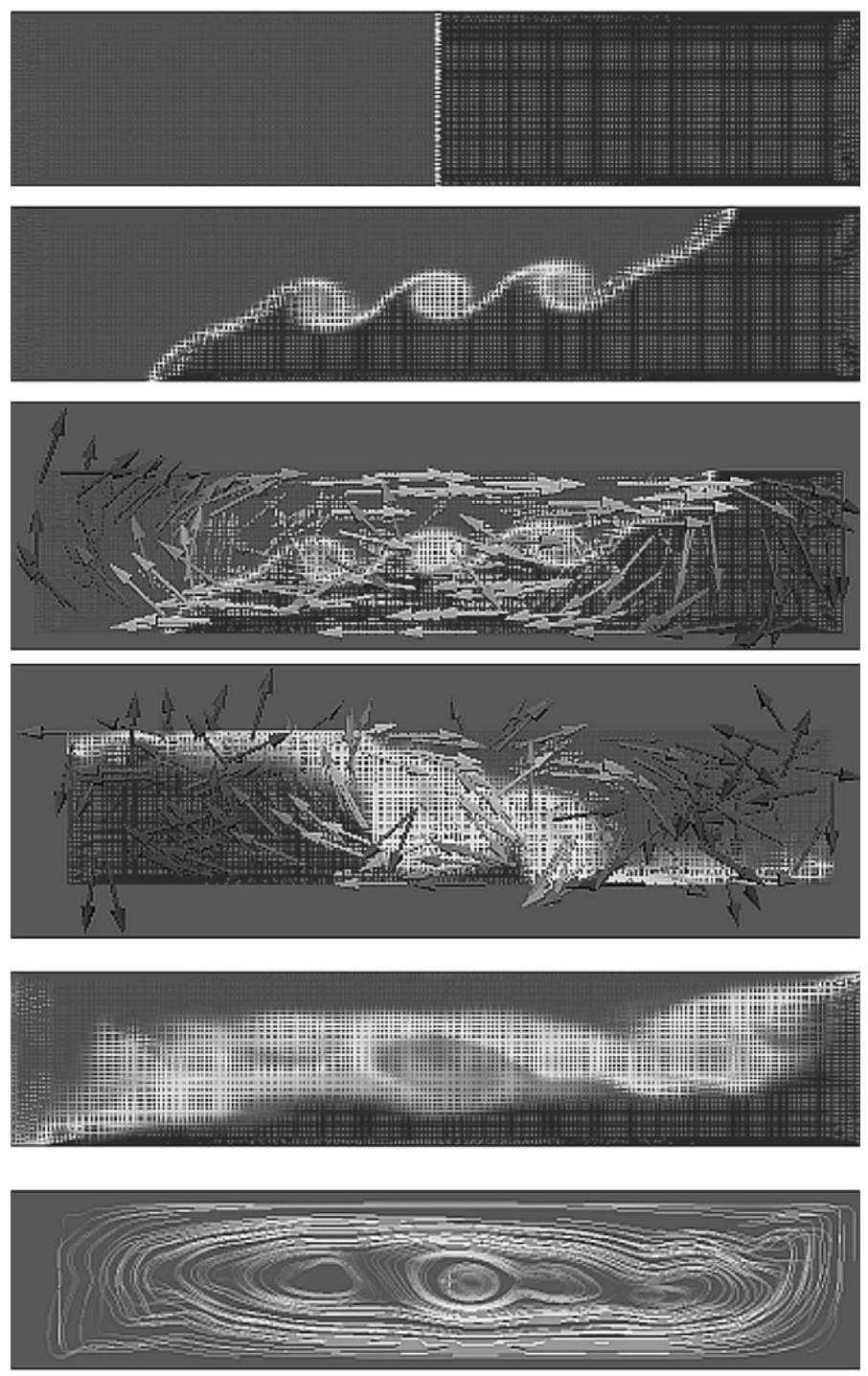

Figure 8. Mixing two fluids of various diffusion coefficients - OpenFoam simulation

\section{Protein Simulation and the MD Method}

Molecular dynamics (Allen, 2004; Frenkel et al., 2001) typically considers the reciprocal interaction between the system of molecules or atoms. It is commonly assumed that the analyzed forces are potential, i.e. they can be expressed as the derivatives of potential energy, and central, i.e. their value depends only on the distance from the source of the force. Fur- 
thermore, we can distinguish long-range forces (electrostatic and gravitational) and short-range forces (vanishing faster than the inverse of a square of the molecule distance), which result from the Lennard-Jones potential. By confining the scope of analysis to the latter only and using $\mathbf{r}_{i}, \vec{v}_{i}, r_{i j}$ to denote, respectively, location and velocity of molecule $i$ as well as the distance between molecules $i$ and $j$, we learn that force $\vec{f}_{i j}=\vec{f}\left(r_{i j}\right)$ is equal to

$$
\vec{f}_{x}\left(r_{i j}\right)=-\frac{x_{i}-x_{j}}{r_{i j}} \frac{d U_{L J}}{d r_{i j}}=48\left(x_{i}-x_{j}\right)\left(\frac{1}{r_{i j}^{14}}-\frac{1}{2 r_{i j}^{8}}\right) .
$$

The overall force acting on molecule $i$ is $\vec{F}_{i}=\sum_{j \neq i} \vec{f}_{i j}$, so the acceleration $\vec{a}_{i}$ of this molecule is calculated as $\vec{a}_{i}=\vec{F}_{i} / m_{i}$. In molecular dynamics, we normally analyze volumetric properties, which are independent from the surface. The surface influence is estimated through the proportion of the number of molecules at the surface to the sum of all molecules that are being examined. In real systems, the number of system molecules amounts to $10^{23}$, thus making the influence of the surrounding surface negligible. However, in algorithmic practice, we usually consider the number of molecules in the $10^{3}-10^{6}$ range, which makes the influence of the surface unnegligible. In order to eliminate them, we can apply the periodic boundary conditions - molecules are typically restricted with a hexahedron, and we assume that the space is filled with an infinite number of such hexahedrons. Once the number of molecules is increased to $10^{9}$, periodic boundary conditions are no longer necessary the computing power of today's computers allows that. MD plays a vital role in computer-aided drug design (CADD). This stems from the fact that MD simulations can reproduce the dynamics of multiple biological processes, such as the creation of enzyme (receptor)-inhibitor (ligand) complexes, or characterize conformational changes in activated receptor molecules with high precision (Jarmuła, 2013; Tautermann et al., 2015; Zhao et al., 2015).

\section{Protein Simulation}

The physiologic environment of proteins is too complex for the computing abilities of the MD method, therefore proteins are usually magnified by placing them in an aqueous solution. The simulation process typically begins with sampling the protein structure in the PDB format (Protein Data Bank). An example of such a simulation in the Gromacs environment (www.gromacs.org) is summarized in the Figures 9 and 10. 
Wojciech Kowalewski et al.

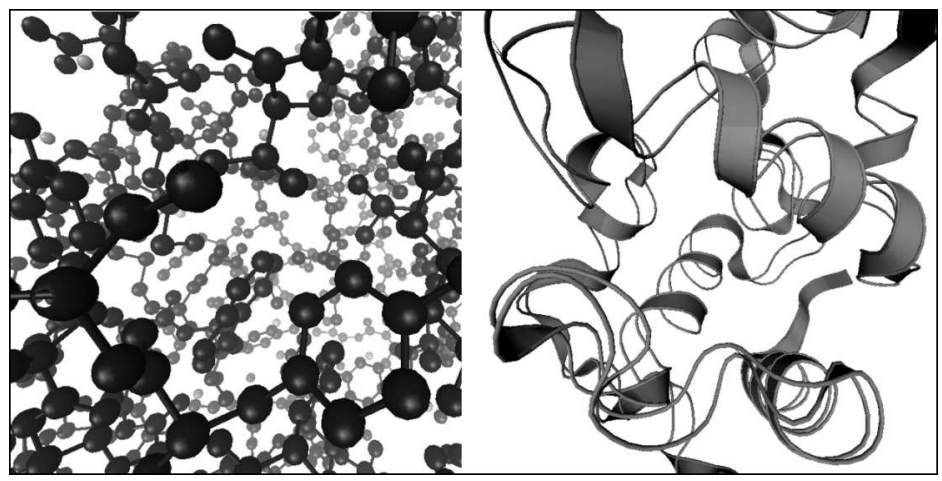

Figure 9. The structure of the orthorhombic form of hen egg-white lysozyme (viewer - VMD: CPK and Ribbons modes); source file: 1AKI.PDB http://www.rcsb.org/

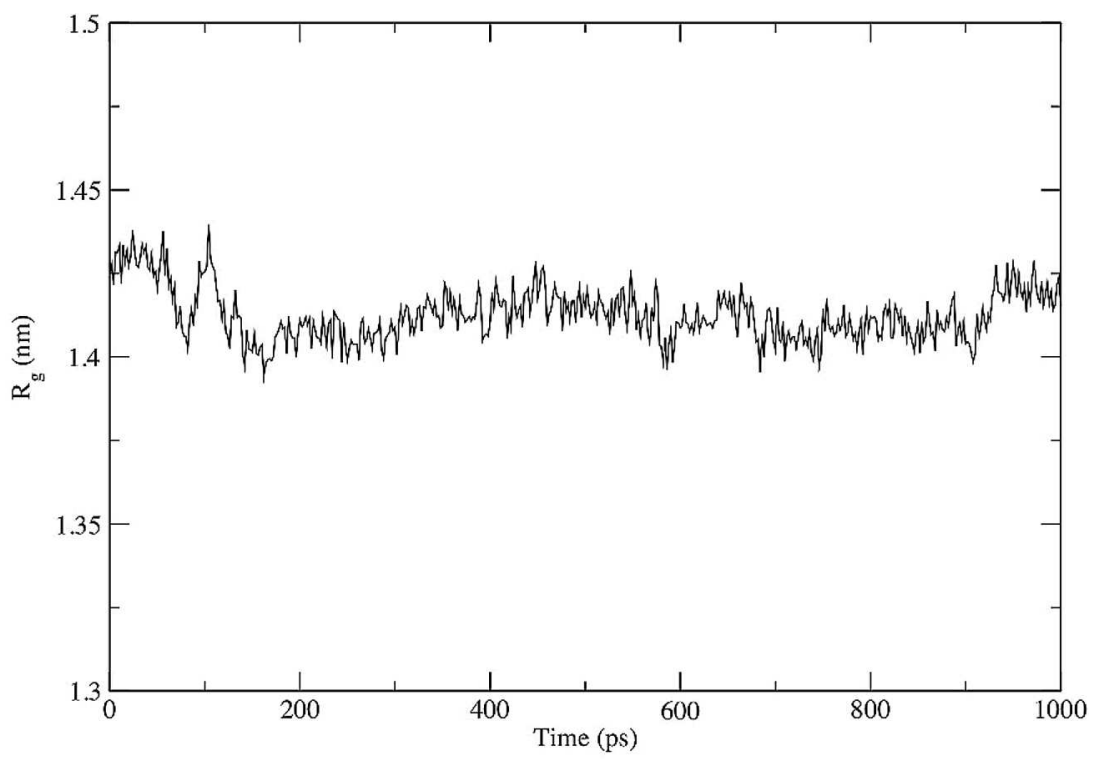

Figure 10. The radius of gyration of a protein - a measure of its compactness; source - Gromascs simulation based on Lemkul (2013)

\section{CFD Analysis}

The above-discussed considerations are summarized by the further specification of the step-by-step procedure for typical problems related to researching fluid behaviors - these actions are recapitulated within the notion 
of the CFD analysis. The CFD analysis is usually divided into 3-7 stages. Some of them must be conducted repeatedly in order to acquire the results of desired quality (Marić et al., 2014; Versteeg et al., 2007). In this paper, the 7 -stage process is presented.

1. Formulation of the problem. The initial stage of the CFD analysis has significant influence over consecutive stages. It usually requires cooperation between an expert from the target field and a CFD specialist. Due to the complexity of even the most fundamental problems of fluid mechanics, this stage should result in the maximal simplification of the final set of problem parameters, permissible without considerable losses in the accuracy of numerical calculations at consecutive stages. For instance, the decision must be made whether these fluids can be considered as incompressible and/or non-turbulent.

2. Mathematical model. This stage may be more or less complex, depending on whether the theoretical model of the analyzed problem has already been researched or still needs to be researched. If the latter is the case, it will be necessary to employ a mathematician or a theoretical physicist, who must collaborate with an expert from the target field in order to construct a correct theoretical model. Still, even if the theoretical model exists, it remains crucial that the CFD specialist understands its significant elements since his/her role at this stage consists of selecting an existing numerical algorithm or developing a new one (or alternatively modifying an existing one), if there are no such algorithms or none are suitable. CFD algorithms are usually developed for the type of equation or a group of equations that may be employed in a wider spectrum than just a single analyzed issue. In very general terms, this stage may be summarized as a two-part process (Price, 2006): first, we choose the coordinate system that is adequate for the analyzed problem, and then we reformulate behavioral principles in the language of that system.

3. Construction of the discretizing grid. The necessity of applying numerical methods in fluid dynamics implies the obligation to discretize the domain of the active field. For example, in grid methods of the mesoscopic model, two distinct approaches are distinguished (Figure 11):

- Lagrangian model - the grid spans the region of the fluid and moves along with it - by tracking its elements, we can follow the paths of respective fluid molecules

- Eulerian model - the grid spans the entire analyzed space and is fixed - we can track its nodes by examining which elements of the fluid are currently located in a given node. In this situation, it is more difficult to investigate trajectories of fluid molecules. 
Wojciech Kowalewski et al.

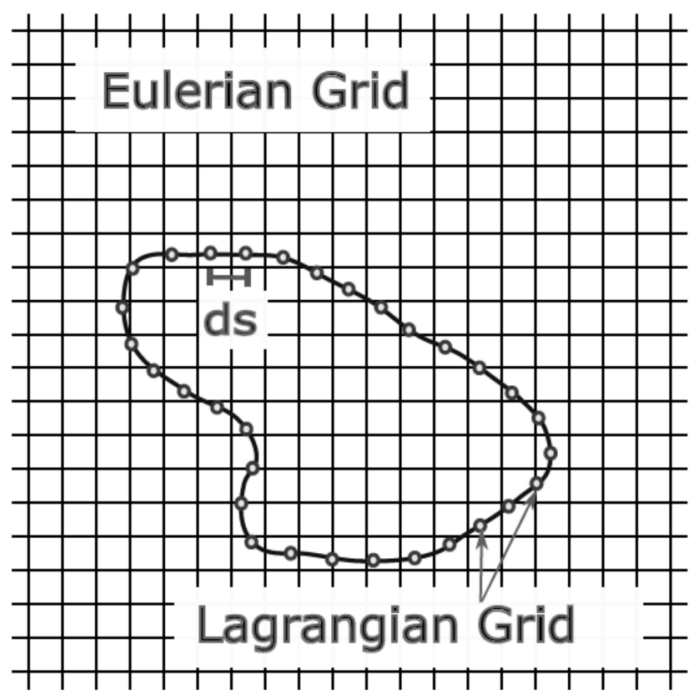

Figure 11. The static Eulerian grid, spread over the fluid domain, and the moving Lagrangian grid with the ds resolution, defined on the boundary of the fluid (based on de Lima et al., 2007: Figure 1)

Depending on the scene geometry and the topology of the objects contained therein, the process of generating grids may prove very complicated. In general, structured and nonstructured models are chosen for such grids. Both options allow adaptive compression of grids in certain subregions, while the latter enables the definition of grids with non-trivial topology.

\section{Calculation of boundary and initial conditions in grid points.} Due to the significant density of discretizing grids, this stage typically requires the employment of additional programs, which are used to prepare files with values that are present in the assumed equations of model parameters at grid boundaries as well as values of the entire field at the moment $t=0$.

\section{Numerical solving of equations of the assumed mathematical model.}

6. Analyses of the achieved solution. At this stage, solutions that are saved in files are usually visualized in different ways with the aid of special tools. Furthermore, the same or other tools allow us to generate additional information concerning the analyzed problem.

7. Critical discussion of the results. The final stage should include the verification of the end results, which is usually done by comparison with values measured in natural conditions. Broadly speaking, these measurements may be found difficult and expensive to make. 
Allen, M. P. (2004). Introduction to Molecular Dynamics Simulation. In N. Attig, K. Binder, H. Grubmüller, \& K. Kremer (Eds.), Computational Soft Matter: From Synthetic Polymers to Proteins, Lecture Notes, NIC Series Volume 23 (pp. 1-28). Jülich: John von Neumann Institute for Computing (NIC).

Chandran, K. B., Rittgers, S. E., \& Yoganathan, A. P. (2012). Biofluid Mechanics: The Human Circulation (Second Edition). Boca-Raton: CRC Press.

de Lima e Silva, A. L. F., da Silva, A. R., \& da Silveira-Neto, A. (2007). Numerical simulation of two-dimensional complex flows around bluff bodies using the immersed boundary method. Journal of the Brazilian Society of Mechanical Sciences and Engineering, 29(4), 379-387.

Frenkel, D. \& Smit, B. (2001). Understanding Molecular Simulation: From Algorithms to Applications. Computational Science. San Diego, San Francisco, New York, Boston, London, Sydney, Tokyo: Academic Press.

Jarmuła, A. (2013). Dynamika molekularna: charakterystyka podstawowa, metody i zastosowanie w projektowaniu leków. Retrieved from http://www.ebiotech nologia.pl/Artykuly/Dynamika-molekularna-charakterystyka-podstawowa metodyi-zastosowanie--w-projektowaniu-lekow/

Lemkul, J. A. (2013). GROMACS Tutorial, Lysozyme in Water. Retrieved from http://www.bevanlab.biochem.vt.edu/Pages/Personal/justin/gmxtutorials/ lysozyme/

Marić, T., Höpken, J., \& Mooney, K. (2014). The OpenFOAM Technology Primer. Sourceflux UG.

Pozrikidis, C. (2010). Computational Hydrodynamics of Capsules and Biological Cells. Series: Chapman \& Hall/CRC Mathematical and Computational Biology (Book 35). Boca Raton: CRC Press.

Price, J. F. (2006). Lagrangian and Eulerian Representations of Fluid Flow: Kinematics and the Equations of Motion. Retrieved from http://www.whoi.edu/ science/PO/people/jprice/class/ELreps.pdf

Rubenstein, D., Yin, W., \& Frame, M. D. (2015). Biofluid Mechanics: An Introduction to Fluid Mechanics, Macrocirculation, and Microcirculation. Biomedical Engineering. Academic Press Series in Biomedical Engineering.

Spagnolie, S. E. (Ed.). (2015). Complex Fluids in Biological Systems: Experiment, Theory, and Computation. Biological and Medical Physics, Biomedical Engineering. New York: Springer.

Spiegelman, M. (2000). Myths \& Methods in Modeling. Retrieved from http://www. ldeo.columbia.edu/ mspieg $/ \mathrm{mmm} /$ course.pdf

Tautermann, C. S., Seeliger, D., \& Kriegl, J. M. (2015). What can we learn from molecular dynamics simulations for GPCR drug design? Computational and Structural Biotechnology Journal, 13, 111-121. 
Wojciech Kowalewski et al.

Tu, J., Inthavong, K., \& Ahmadi, G. (2013). Computational Fluid and Particle Dynamics in the Human Respiratory System. Biological and Medical Physics, Biomedical Engineering. New York: Springer.

Tu, J., Inthavong, K., \& Wong, K. K. L. (2015). Computational Hemodynamics Theory, Modelling and Applications. New York: Springer.

Versteeg, H., \& Malalasekera, W. (2007). An Introduction to Computational Fluid Dynamics: The Finite Volume Method (2nd Edition). England: Pearson.

Zhao, H., \& Caflisch, A. (2015). Molecular dynamics in drug design. European Journal of Medicinal Chemistry, 91, 4-14. 